Meta

Journal des traducteurs

Translators' Journal

\title{
Notes pour une histoire de la traduction pharmaceutique
}

\section{Henri Van Hoof}

Volume 46, numéro 1, mars 2001

Traduction médicale et documentation / Medical translation and documentation

URI : https://id.erudit.org/iderudit/004551ar

DOI : https://doi.org/10.7202/004551ar

Aller au sommaire du numéro

Éditeur(s)

Les Presses de l'Université de Montréal

ISSN

0026-0452 (imprimé)

1492-1421 (numérique)

Découvrir la revue

Citer cet article

Van Hoof, H. (2001). Notes pour une histoire de la traduction pharmaceutique. Meta, 46(1), 154-175. https://doi.org/10.7202/004551ar

\section{Résumé de l'article}

Cet article retrace l'histoire de la rédaction et de la traduction des ouvrages pharmaceutiques des origines (troisième millénaire avant Jésus-Christ) à aujourd'hui tant en Orient qu'en Occident, en s'attardant plus particulièrement sur les apports orientaux et arabes à la pharmacologie occidentale à partir du Moyen Âge. 


\title{
Notes pour une histoire de la traduction pharmaceutique
}

\author{
HENRI VAN HOOF \\ Institut libre M. Haps, Bruxelles, Belgique
}

\section{RÉSUMÉ}

Cet article retrace l'histoire de la rédaction et de la traduction des ouvrages pharmaceutiques des origines (troisième millénaire avant Jésus-Christ) à aujourd'hui tant en Orient qu'en Occident, en s'attardant plus particulièrement sur les apports orientaux et arabes à la pharmacologie occidentale à partir du Moyen Âge.

\section{ABSTRACT}

This article retraces the history of writing and translating pharmaceutical works from the early beginnings (3000 B.C.) to present day, in both the Orient and the West, with particular attention to oriental and arabic contributions to pharmacology in the West, dating back to the Middle Ages.

\section{MOTS-CLÉS/KEYWORDS}

traduction pharmaceutique, histoire de la traduction, chronologie, pharmacognosie

Il n'existe aucune certitude sur les origines de la pharmacie. Certes, on peut imaginer que les hommes ont, depuis les temps les plus anciens, cherché des remèdes à leurs souffrances, souvent aidés en cela par l'observation des animaux. Virgile ne rappellet-il pas que ce sont les cerfs et les chèvres sauvages qui leur ont appris l'emploi de l'origan et des vulnéraires? Ces remèdes empiriques passèrent très vite aux mains de guérisseurs, dont l'emprise était d'autant plus grande qu'ils prétendaient tirer leurs recettes d'une source surnaturelle. De ce fait, l'art de guérir fut bientôt associé aux divinités, à l'incantation magique, à la prière. Ce caractère théurgique se trouve confirmé dans certains livres de l'Ancien Testament, notamment dans le Lévitique (14, 52-57), que l'exégèse place entre le $\mathrm{x}^{\mathrm{e}}$ et le $\mathrm{vI}^{\mathrm{e}} \mathrm{s}$. avant notre ère mais dont la substance remonterait à l'époque de Moïse (XIII ${ }^{\mathrm{e}}$ s. av. J.-C.). On peut y lire: «Le sacrificateur prendra [...] deux oiseaux, du bois de cèdre, du cramoisi et de l'hysope. Il égorgera l'un des oiseaux sur un vase de terre, sur de l'eau vive. Il prendra le bois de cèdre, l'hysope et le cramoisi et l'oiseau vivant, et il en fera sept fois l'aspersion sur la maison $[\ldots]$. Telle est la loi pour toute plaie de lèpre et pour la teigne $[\ldots]$, pour les tumeurs, les dartres et les taches ». Et le Livre des Rois (II, 20,7), daté du vi ${ }^{\mathrm{e}}$ s. av. J.-C. relate que: «En ce temps-là, Ezéchias fut malade à la mort [...]. Le prophète Isaïe dit: "Prenez une masse de figues." On la prit et on l'appliqua sur l'ulcère. Et Ézéchias guérit.»

\section{Les temps prégaléniques}

Les versets bibliques ne sont pas les allusions les plus anciennes à l'art de guérir, bien qu'il soit difficile de décider à quel document attribuer la palme de l'ancienneté. On

Meta, XLVI, 1, 2001 
a cru longtemps qu'elle revenait au Pen-ts'ao (Traité de matière médicale) de l'empereur Chen Nong, qui l'aurait composé au $\mathrm{xxxvI}^{\mathrm{e}} \mathrm{s}$. avant notre ère. Mais Chen Nong est un empereur mythique et l'ouvrage, qui contient trois cent soixante-cinq drogues minérales, végétales ou animales, aurait en réalité été rédigé sous les Han (v. le $\mathrm{I}^{\mathrm{er}} \mathrm{s}$. av. J.-C.) par un auteur inconnu se cachant sous un pseudonyme légendaire et exploitant des sources antérieures ne remontant pas au-delà du $\mathrm{IV}^{\mathrm{e}} \mathrm{s}$. av. J.-C. Par la suite, chaque grande époque se dota de son compendium de matière médicale: Chang Ching-ching ( $\mathrm{II}^{\mathrm{e}} \mathrm{s}$.), Tang pen-ts'ao ( $\mathrm{vII}^{\mathrm{e}} \mathrm{s}$.), Pen-ts'ao che-yi (viII ${ }^{\mathrm{e}} \mathrm{s}$.), Kai-pao pen-ts'ao ( $\mathrm{x}^{\mathrm{e}} \mathrm{s}$.), Chao-Hing pen-ts'ao ( $\mathrm{xiI}^{\mathrm{e}} \mathrm{s}$.), etc.

La plus vieille pharmacopée serait 1'une des nombreuses tablettes cunéiformes découvertes à Nippour (Mésopotamie) au début du siècle; gravée à la fin du III $^{\mathrm{e}}$ millénaire avant notre ère, elle mentionne une demi-douzaine de remèdes, la plupart tirés du règne végétal mais certains contenant des substances animales ou minérales. D'autres tablettes nous apprennent que, chez les peuples de la Mésopotamie ancienne, l'exercice de la thérapeutique était surtout de nature sacerdotale.

En Égypte aussi, lorsqu'on étudie la période entre 1600 et 1200 av. J.-C., on constate que la science pharmaceutique était étroitement liée à l'histoire du sacerdoce. Le culte d'Isis, particulièrement, était célèbre pour les traitements que la déesse révélait la nuit aux malades transportés dans ses temples. La préparation des remèdes s'effectuait à l'intérieur même des temples par des spécialistes préposés à cette tâche: les prêtres pastophores. Dans le papyrus découvert par l'égyptologue allemand Georg Ebers, document classique de la pharmacologie égyptienne du début de la XvıII dynastie (1580-1320 av. J.-C.), se trouvent réunis plus de sept cents substances médicinales - plantes, minéraux, sécrétions animales - et plus de huit cents recettes pour des affections allant de la morsure de crocodile jusqu'aux douleurs dans les ongles des orteils. Le papyrus Hearst, découvert en 1899 et datant du règne de Thoutmosis III (1504-1450 av. J.-C.), ainsi que le papyrus de Londres, datant du règne de Toutankhamon (1352-1320 av. J.-C.), sont deux autres recueils de remèdes et de recettes qui trahissent la connaissance de formes pharmaceutiques déjà nombreuses et compliquées.

Parmi ces documents, mis au jour et traduits seulement aux $\mathrm{XIX}^{\mathrm{e}}$ et $\mathrm{XX}^{\mathrm{e}} \mathrm{s}$., $\mathrm{y}$ en a-t-il auxquels d'autres civilisations anciennes auraient eu accès? Y en a-t-il qui auraient pu faire l'objet de traductions en sumérien, en sanskrit? Si rien ne permet de l'affirmer, on sait pourtant que les thérapeutes de Mésopotamie avaient des contacts avec d'autres cultures. Vers 1300, par exemple, le Babylonien Raba-sha-Mardouk donnait des consultations à la cour du roi hittite au milieu de médecins égyptiens. À l'inverse, l'Égypte, qui dès la IV e dynastie (2575-2465 av. J.-C.) et jusqu’à la VI ${ }^{e}$ (2325-2155 av. J.-C.) entretint des rapports suivis avec la Babylonie, aurait-elle pu connaître et traduire des pharmacopées de la langue akkadienne? Rien n'est moins sûr, même si Hérodote, qui pourtant chantait l'Égypte comme la patrie de l'art de guérir, assurait dans le même temps que les Assyriens, les Chaldéens et les Indiens furent les premiers à composer des remèdes.

En Inde, l'art pharmaceutique était aux mains des brahmanes, caste sacerdotale dépositaire de la tradition védique. C'est l'Atharva-véda (v. 1500 av. J.-C.), la quatrième et dernière des Védas, qui traite de la matière médicale. Elle contient des chapitres sur les toniques (Hasayana), les aphrodisiaques (Vajikarana tantra) et la toxicologie (Agada tantra). Moins anciens, les ouvrages les plus célèbres de la médecine brahmanique 
sont attribués à des auteurs de notre ère: Caraka ( $\mathrm{II}^{\mathrm{e}} \mathrm{s}$.) et Susruta $\left(\mathrm{IV}^{\mathrm{e}} \mathrm{s}\right.$.). La Samhitâ (Collection) de Caraka comprend une première partie (Sutrasthana) consacrée à la pharmacologie et répertorie plus de cinq cents remèdes; selon Ibn an-Nadim ( $\mathrm{x}^{\mathrm{e}} \mathrm{s}$.), médecin de Bagdad, elle aurait été traduite d'abord en persan et, plus tard, en arabe par un certain Abd Allah Ibn Ali. La Samhitâ de Susruta, qui mentionne plus de sept cent cinquante remèdes, aurait été traduite en pehlvi sous les Sassanides (226-651) et en arabe au viII ${ }^{\mathrm{e}}$ s. par le même auteur d'une version du Livre des poisons de Cânakya (ou Sânâq), médecin et ministre du roi indien Candragupta (315-291 av. J.-C.). Que l'art pharmaceutique indien ait franchi les frontières de l'Inde, on n'en peut douter. Une première preuve en est fournie par le Livre des racines, le plus ancien des textes médicaux tibétains, traduit du sanskrit au viII ${ }^{e}$ s., découvert par le sinologue allemand Ferdinand Lessing. Et, par ailleurs, des remèdes indiens figurent dans les traités chinois de matière médicale dès le début de notre ère.

Dans le Zend Avesta ( $\mathrm{vi}^{\mathrm{e}}$ s. av. J.-C.), livre sacré des anciens Perses, comme dans l'Atharva-Véda, la matière médicale avoisine la philosophie et l'enseignement religieux. Venant après les invocations et la théomédecine, elle décrit des produits tirés des trois règnes. Une traduction officielle du zend en pehlvi en fut faite sous les Sassanides déjà; des versions sanskrites ont suivi aux $\mathrm{XII}^{\mathrm{e}}$ et $\mathrm{XIII}^{\mathrm{e}} \mathrm{s}$.

Ce rapide survol des civilisations anciennes a permis de constater que les remèdes dont disposaient les premiers thérapeutes trouvaient leur substance dans les règnes végétal, animal et minéral, mais qu'il est malaisé d'en déduire ce qui, dans cette thérapeutique, est original ou emprunté à une culture antérieure ou voisine soit par la transmission orale, soit par la traduction.

\section{Du Corpus hippocraticum à la pharmacie galénique}

À ses débuts, la thérapeutique grecque se ressentit des influences mésopotamiennes et égyptiennes et la littérature homérique nous en décrit quelques traits: pratiques magiques, breuvages enchantés, poudres merveilleuses. C'est vers le $\mathrm{v}^{\mathrm{e}} \mathrm{s}$. avant notre ère qu'elle se sépare de l'emprise théurgique: Hippocrate crée une médecine libérée de l'empirisme et fondée sur l'observation clinique. Médecin ambulant, il était amené aussi à dispenser des remèdes qu'il préparait parfois lui-même. Le Corpus hippocraticum compilé après sa mort - car il n'a pas laissé d'écrits de sa main contient des prescriptions médicamenteuses qui font appel aux matières des trois règnes et se présentent sous des formes pharmaceutiques déjà très élaborées. Les remèdes à usage interne sont soit liquides (potions, décoctions, infusions), soit solides (pilules, collyres, pastilles, suppositoires - héritage direct de la pharmacie égyptienne); les remèdes à usage externe comprennent des fumigations, des gargarismes, des onguents, des cataplasmes et des lavements.

Après la conquête de l'Égypte par les Grecs, la ville d'Alexandrie, fondée par Alexandre le Grand, devint rapidement le nouveau centre du savoir sous Ptolémée I ${ }^{\text {er }}$ Soter (360-280 av. J.-C.). L'art médico-pharmaceutique y connut un nouvel essor et donna d'abord naissance à l'école dogmatique - représentée par Hérophile, créateur de la matière médicale - puis à l'école empirique - illustrée par Sérapion, inventeur de la théorie polypharmaque, et Héraclite de Tarente, promoteur de l'usage de l'opium, qui entra dès lors dans la confection de tous les remèdes polypharmaques. Nicandre ( $\mathrm{II}^{\mathrm{e}}$ s. av. J.-C.) développa les théories de ses prédécesseurs et les 
consigna dans deux poèmes didactiques: la Theriaca, qui décrit les médicaments à usage interne pour combattre les empoisonnements par morsure animale, et l'Alexipharmaca, qui décrit les toxiques des trois règnes, les symptômes d'empoisonnement et les moyens pour les neutraliser. Les écoles d'Alexandrie ont encore enrichi l'arsenal pharmaceutique de remèdes spéciaux, comme le soufre dans le traitement de la teigne. C'est alors aussi que, pour la première fois, la médecine se serait, selon Celse, divisée en trois branches: la diététique (maladies et régimes), la chirurgie (interventions du fer et du feu) et la pharmaceutique (médecine médicamenteuse). Cette classification passa telle quelle à l'empire romain.

À l'origine, la médecine romaine fut elle aussi théurgique mais, comme Alexandrie, Rome accueillit par la suite une immigration de médecins grecs inaugurée par Aesclépiade de Bythinie, qui remit en honneur les principes hippocratiques et simplifia la thérapeutique en privilégiant les remèdes externes pour réduire les abus de la polypharmacie. À leur suite, s'installèrent des médecins égyptiens et juifs. Les conditions paraissaient ainsi remplies pour voir éclore une activité de traduction qui a fait défaut à la Grèce trop imbue de la supériorité de sa langue. Mais dans quelle mesure le De re medica $\left(\mathrm{I}^{\mathrm{er}} \mathrm{s}\right.$.), dans lequel Celse résume la médecine alexandrine, est-il une compilation, une adaptation ou une traduction libre? Dans le cinquième et dans une partie du sixième livre, qui concernent la pharmacie, Celse fait la distinction entre les pratiquants de la pharmaceutique (pharmaceutes), les marchands qui vendent des médicaments tout préparés (pharmacopoles), les pileurs de drogues (pharmaceutribes), les marchands de plantes communes (herbarii) et les droguistes (seplasiarii). Pline l'Ancien (23-79), dans son Histoire naturelle, réserve quinze des trente-sept livres aux remèdes tirés des plantes. L'ouvrage le plus important, car entièrement consacré à la matière médicale, est le Peri hulès iatrikhès, rédigé sous Néron par le médecin et botaniste grec Dioscoride ( $\mathrm{I}^{\mathrm{er}} \mathrm{s}$.). Ses cinq livres décrivent plus de six cents matériaux végétaux (plantes, fleurs, feuilles, écorces, racines, sucs), mais aussi quelques minéraux et produits chimiques (acétate de plomb, antimoine, sels de cuivre). Connu de l'Italie byzantine, le texte grec fut traduit en latin vers le $\mathrm{vI}^{\mathrm{e}} \mathrm{s}$. en Italie du Sud ou en Afrique du Nord; une version arabe vit le jour à Bagdad au $\mathrm{IX}^{\mathrm{e}} \mathrm{s}$. et se répandit dans l'Espagne mauresque dès le $\mathrm{x}^{\mathrm{e}} \mathrm{s}$. Le point culminant de la médecine romaine fut atteint avec Galien, médecin grec également, qui pratiqua les diverses disciplines de l'art de guérir, y compris la pharmacie, préparant lui-même ses remèdes dans sa boutique de la Voie Sacrée. Auteur de plus de cinq cents ouvrages, dont plusieurs traitant de remèdes et prescriptions, de médicaments simples et composés, il est surtout le fondateur de la branche la plus spécifiquement pharmaceutique de l'art, celle qui s'occupe des formes d'administration — la pharmacie dite galénique. Sans doute parce que tous les hommes de l'art savaient le grec, partie intégrante du bagage intellectuel de l'homme cultivé, Rome n’a laissé que peu de traductions médicales et aucune dans le domaine pharmaceutique. Après Galien (131-210), l'Empire romain entra en décadence. Le peuple versa dans la magie et le mysticisme, ce qui favorisa l'implantation de religions nouvelles et, finalement, l'avènement du christianisme lorsque la liberté religieuse fut promulguée en 313 par l'empereur Constantin, qui fonda sur le site de l'antique Byzance la capitale d'un empire romain d'Orient. 
158 Meta, XLVI, 1, 2001

\section{Le monde byzantin et l'intermède nestorien}

Dans le monde byzantin, dominé par l'idéal chrétien, l'art de guérir redevint théurgique. La médecine galénique fut réprouvée et la littérature médico-pharmaceutique byzantine n'a légué que des compilations auxquelles s'attachent les noms d'un Oribase de Pergame ( $\mathrm{IV}^{\mathrm{e}} \mathrm{s}$.), auteur d'un Euporista ou les Remèdes faciles à préparer, d'un Paul d'Égine ( $\mathrm{viI}^{\mathrm{e}} \mathrm{s}$.), auteur d'un ouvrage sur les Remèdes simples, d'un Alexandre de Tralles ( $\mathrm{vi}^{\mathrm{e}} \mathrm{s}$.), etc.

Sous l'empereur Théodose II, se produisit un événement dont les conséquences pour l'histoire de la traduction médicale, et pharmaceutique en particulier, revêtent une importance capitale. Nestorius (380-451), patriarche de Constantinople, fut condamné pour hérésie par le concile d'Éphèse et exilé en 431. Avec ses adeptes, il s'installa d'abord à Édesse (l'actuelle Urfa, en Turquie), puis à Nisibe (actuellement Nizip, en Turquie), où ils créèrent des écoles de médecine. Chassés par les persécutions religieuses, ils se réfugièrent en Perse, dans le Khorassam, pour se fixer dans la ville de Gondashepur, qui attachera son nom à leur établissement d'enseignement le plus fameux. Connaissant les écrits d'Hippocrate, de Galien, de Dioscoride, ils en recherchèrent des manuscrits pour les traduire en syriaque, en persan, en hébreu. Parmi les traducteurs, on relève, pour le domaine pharmaceutique, le prêtre-médecin chrétien Sergius de Rhésine ( vII $^{\mathrm{e}}$ s.), qui a mis en syriaque les livres LXVII et LXVIII des Médicaments simples de Galien.

Lorsque les Arabes envahirent la Perse en 651, ils découvrirent les manuscrits nestoriens, mais la barrière des langues s'opposa à ce premier contact avec la médecine grecque. Aussi, dès la seconde moitié du viI ${ }^{\mathrm{e}} \mathrm{s}$., les califes abbassides décidèrent de les faire traduire en arabe. On pourrait dès lors considérer que l'héresie nestorienne est à l'origine de la médecine et de la pharmacie arabes, et les traductions arabes à leur tour assureront la liaison entre l'Antiquité et le Moyen Âge occidental.

\section{La passerelle arabe}

Après que la foi guerrière des Arabes eût porté l'islamisme en Asie jusqu'à l'Indus et, en Europe, des rives de la Méditerranée jusqu'à l'Atlantique, le pouvoir arabe se concentra dans les califats oriental de Bagdad (762) et occidental de Cordoue (756). Jamais, dans l'histoire, la traduction n'a joué un rôle aussi important que celui qui lui fut dévolu au début de l'expansion islamique, et les médecins nestoriens y tinrent encore une place prépondérante.

\section{Dans le califat oriental}

Jurgis Bakhtishu, médecin-chef de l'hôpital de Gondashepur, appelé à Bagdad par le calife al-Mansur (754-775), fut chargé par celui-ci de traduire de nombreux ouvrages grecs; il aurait ainsi mis en syriaque les Remèdes correspondant aux maladies de Galien. Une école de traduction fut installée à Bagdad par le calife al-Mamun (786-833) sous l'appellation de Bayt alhikma (Maison de la Sagesse) et confiée à la direction de Yuhanna Ibn Masawaih (776-855), mieux connu sous le nom latinisé de Jean Mésué ou Mésué l'Ancien et issu, lui aussi, d'une famille de médecins de Gondashepur. Mésué non seulement organisa la traduction en arabe d'ouvrages médicaux grecs, 
syriaques et persans, mais encore traduisit-il lui-même les versions syriaques de Galien réalisées par Sergius de Rhésine. Il a de plus écrit des œuvres personnelles, parmi lesquelles un Traité sur les substances simples aromatiques et une Pharmacopée générale, dont il n'existe qu'une traduction latine intitulée De consolatione medicinarum simplicium $\left(\mathrm{xI}^{\mathrm{e}} \mathrm{s}\right)$.

Le plus grand élève de Mésué fut sans conteste Hunayn Ibn Ishaq (dit Johannitius, 808-873), chrétien nestorien, médecin et philosophe. Fils d'apothicaire, il apprit le grec au cours de nombreux voyages, alors qu'il savait déjà parfaitement le syriaque et l'arabe. Revenu à Bagdad, il se lança dans une prodigieuse activité de traduction avec l'aide, notamment, de son fils Ishaq et de son neveu Hubaysh al-Asam. De cette collaboration naquirent une centaine d'œuvres. Bien qu'il traduisît lui-même du grec ou du syriaque en arabe, Hunayn préférait le plus souvent laisser l'arabisation à ses assistants. Son inclination personnelle allait à la traduction en syriaque, langue dans laquelle on lui doit, du point de vue pharmaceutique, des versions du Corpus hippocraticum, les traités Les remèdes simples, Les médicaments faciles à obtenir, Les remèdes correspondant aux maladies de Galien, divers écrits de Paul d'Égine et d'Oribase, que son fils Ishaq et surtout Hubaysh mirent ensuite en arabe, du Peri hulès iatrikhès (Matière médicale) de Dioscoride, dont la version arabe de son collaborateur Istafan Ibn Basil devint pour longtemps un livre de référence pour le monde islamique. À l'actif de son neveu, on peut ajouter encore la traduction du Peri khraseos khai dynameos to apleon pharmakhon de Galien, dont une version avait été donnée déjà au début du $\mathrm{Ix}^{\mathrm{e}} \mathrm{s}$. par al-Bitriq.

Parmi les autres traducteurs arabes qui se sont penchés sur des textes pharmaceutiques, on peut citer Yahya Ibn Ibrahim, membre de l'équipe d'Hunayn, qui mit en arabe la version syriaque établie par Hunayn de La force des laxatifs de Galien et celle des Remèdes correspondant aux maladies, du même auteur, faite par Bakhtishu; il y a aussi an-Natili, qui se livra en 985 à une révision de la traduction de la Materia medica de Dioscoride par Hunayn.

On traduisait aussi de l'arabe en persan, comme al-Kasani le fit en 1228 pour La science des drogues médicinales d'al-Biruni (973-1048) en donnant les noms de plantes en grec, en syriaque, en indien, en hébreu, etc., ainsi que de l'arabe en syriaque, comme le médecin chrétien Abul Faradj (dit Bar Hebraeus, 1226-1286) - qui fut aussi l'interprète de Dioscoride - le fit pour le Livre des drogues simples du Cordouan al-Ghafiqi ( $\mathrm{XII}^{\mathrm{e}} \mathrm{s}$.) en donnant les synonymes des plantes en arabe, en berbère et en espagnol. Ces glossaires multilingues mettent en lumière les innombrables difficultés rencontrées par les traducteurs.

Les Arabes, qui empruntèrent beaucoup aux autres, ne s'intéressaient pas qu'aux seuls auteurs grecs. Dès le viII ${ }^{\mathrm{e}}$ s., le calife Harun al-Rachid (766-809) invitait à sa cour le médecin indien Mankah (en sanskrit Manikya) avec mission de mettre en arabe des ouvrages de l'art thérapeutique indien. Mankah traduisit ainsi la SusrutaSamhitâ, le grand classique du $\mathrm{IV}^{\mathrm{e}} \mathrm{s}$., dont la pharmacognosie énumère plus de sept cent cinquante remèdes, et le Livre des poisons de Sânâq (en sanskrit Cânakya); il aurait donné de ce dernier une version persane dont al-Gauhari ( $\mathrm{IX}^{\mathrm{e}} \mathrm{s}$.) assura la version arabe à la demande d'al-Mamun. Un autre classique indien, la CarakaSamhitâ du $\mathrm{II}^{\mathrm{e}}$ s., aurait également été traduite d'abord en persan puis en arabe par un certain Abd Allah Ibn Ali. 
Le califat oriental a produit trois grands noms de l'art médico-pharmaceutique: Rhazès (ar-Rhazi, 865-925), Haly Abbas (al-Magusi, ?-994) et Avicenne (Ibn Sina, 980-1037), tous persans. À côté de son œuvre maîtresse, le Kitab al-Hawi, encyclopédie médicale et pharmacologique qui donne des prescriptions préconisant les drogues dont il a vérifié l'efficacité, Rhazès a aussi laissé un formulaire de médicaments composés (Aqrabadin al-kabir). Le Kitab al-Malaki (Livre royal de l'art et de la médecine) de Haly Abbas, qui sera le premier ouvrage médical arabe traduit en latin, comprend un chapitre sur le traitement par les drogues simples et un autre sur les remèdes composés. Dans son Canon, Avicenne consacre de nombreux chapitres à la description des simples, aux poisons, aux remèdes composés et à la préparation des médicaments, inspirés en grande partie de la traduction du Peri syntheseon pharmakon de Galien, qui a conditionné toute la littérature pharmaceutique arabe.

C'est d'ailleurs par leurs pharmacologues qu'on peut le mieux juger le rôle des Arabes dans le domaine pharmaceutique. Leurs livres portent le nom d'aqrabadin, rendu au Moyen Âge par antidotarium ou dispensatorium. Ishaq al-Kindi (?-870), auteur d'un antidotaire de huit cents remèdes arabes, persans, indiens et égyptiens, a aussi écrit un Kitab fi Marifat (Connaissance des propriétés des médicaments composés) traduit en latin sous le titre De medicinarum compositorum gradibus investigandis libellus; Gabir Ibn Haiyan ( $\mathrm{IX}-\mathrm{x}^{\mathrm{e}} \mathrm{s}$.) est l'auteur d'un Kitab as-Sumum (Livre des poisons); un ouvrage similaire de la première moitié du $\mathrm{x}^{\mathrm{e}} \mathrm{s}$. aurait été dicté en arabe à Ali az-Zayyat par Ibn Wahsiya, traduit d'un original nabatéen de Yarbuqa; le médecin et philosophe Abd al-Latif (1162-1231), de Bagdad, a composé un Kitab fi Usul (Traité de pharmacologie) avec un petit répertoire de trente et une drogues; Aidamir al-Gildaki a signé un Kitab Durrat, compendium de cinq cent soixante-dix noms de drogues végétales, animales et minérales, avec les synonymes en persan, syriaque et byzantin; le médecin et botaniste Ibn al-Baitar (1197-1248), né à Malaga, mais qui exerça en Égypte et en Syrie, s'est signalé par un commentaire sur la Materia medica de Dioscoride et, surtout, par un monumental Kitab al-Djami, ouvrage le plus connu de la pharmacognosie arabe, où sont décrites quelque mille huit cents drogues végétales, cent trente drogues animales et cent quarante-cinq drogues minérales; son élève as-Suwaidi (1204-1292), médecin à Damas et au Caire, est connu pour son Kitab at-Tadkira, recueil de quatre cents remèdes et recettes, et pour son Kitab as-Simat, compendium des noms de plantes médicinales le plus complet de son époque, avec équivalents en grec, syriaque, persan, berbère, castillan et, pour l'arabe, la distinction entre l'usage de Syrie, d'Égypte et d'Espagne.

\section{Dans le califat occidental}

Dans le califat de Cordoue également, l'art médico-pharmaceutique a été dominé par trois grands: Abulcasis (Ibn al-Qasim, 912-961), Averroès (Ibn Rushd, 11261198) et Maïmonide (Ibn Maimun, 1135-1204). Le Kitab at-Tasrif (Exposition des matières) d'Abulcasis consacre une grande partie aux extraits de plantes, aux remèdes simples et à la préparation des remèdes. Le Kitab al-Kulliyat (CEuvres complètes) d'Averroès, que le Moyen Âge occidental latinisera en Colliget, comprend des chapitres sur la pharmacologie et la thérapeutique. Son disciple, le médecin judéo-espagnol et rabbin Maïmonide, a traduit en hébreu le Canon d'Avicenne et composé de nombreux traités, parmi lesquels un Kitab as-Sumum (Livre des poisons) et un Kitab Sahr 
asma al-uqqar (Explication des noms de drogues) de quatre cent cinq rubriques avec équivalents arabes, grecs, syriaques, persans, berbères et andalous. Cette précaution reflète bien les immenses problèmes de communication auxquels est confronté le monde arabe. D'autres illustrations en sont le Kitab al-Talhir d'Ibn Djanah (?-v. 1040), médecin judéo-arabe de Saragosse, qui donne les noms de drogues en six langues (arabe, persan, syriaque, grec, berbère et espagnol), le Kitab al-Mustaini (Traité de pharmacologie) d'Ibn Biklaris (XI-XII ${ }^{e}$ s.), médecin juif d'Almeria, où les noms des drogues simples figurent en six ou sept langues (arabe, persan, syriaque, grec, latin, espagnol et, parfois, en dialecte de Saragosse), le Kitab al-Djami (Recueil des drogues simples) d'al-Idrisi ( ?-1166), qui cite les plantes avec leurs équivalents en six à douze langues (dont le grec, l'hébreu, le turc, le kurde, l'indien et le castillan).

L'empire musulman, de par son extension territoriale considérable, a introduit dans la thérapeutique des médicaments nouveaux provenant de divers pays d'Asie et d'Europe. Ses savants ont enrichi la science pharmaceutique par leur apport personnel sous la forme de préparations nouvelles, d'opérations telles que la distillation et la percolation et, surtout, par l'application de la chimie à la matière médicale. En cela, l'apport du califat occidental ne le cède en rien à celui du califat oriental et, de même, la traduction n'est pas moins présente à Cordoue qu'à Bagdad. En 951, par exemple, le médecin hispano-arabe Hasday Ibn Schaprut (915-970), ministre du calife de Cordoue Abd ar-Rahman III et fondateur de l'académie des sciences de la ville, s'attaquait à une nouvelle version arabe de la Materia medica de Dioscoride sur la base d'un codex grec offert par l'empereur de Byzance. Il fut aidé dans sa tâche par le moine médecin byzantin Nikolaos, dépêché par Constantin VII, et par un autre médecin cordouan, al-Haitam. Au milieu du $\mathrm{x}^{\mathrm{e}}$ s., Ibn Djuldjul, médecin de Cordoue, entreprenait à son tour d'améliorer la traduction du traité de Dioscoride, publiant même une Explication des noms de remèdes simples figurant dans l'auvre de Dioscoride (983); quelque deux cents ans plus tard, Dioscoride bénéficia d'une nouvelle version réalisée par Mihran al-Masihi ( $\mathrm{XII}^{\mathrm{e}}$ s.) sur le syriaque d'Hunayn Ibn Ishaq, après l'échec d'une tentative précédente d'un certain Salim al-Malati mandaté par Nadjm ad-Din Alpi (1154-1176), seigneur de Diyarbakir (Kurdistan).

Tous les classiques de la pharmacognosie arabe, mais aussi toutes les traductions arabes du patrimoine pharmaceutique grec, l'Occident chrétien allait les découvrir lorsque la victoire sur les Maures aurait ouvert l'accès aux riches bibliothèques qu'ils s'étaient constituées.

\section{L'éveil de l'Occident chrétien}

L'Europe occidentale, après avoir joui de la pax romana pendant près de quatre siècles qui l'ont éveillée à la civilisation, retomba dans la barbarie avec la disparition de l'Empire romain d'Occident (476). Livrée au chaos des invasions, elle le fut aussi à celui de l'esprit. L'effort de Charlemagne (742-814), fondateur d'une culture d'inspiration latine d'essence chrétienne, ne brilla que brièvement. Le flambeau fut repris par les monastères, qui concentrèrent la puissance culturelle jusqu'à l'apparition des premières universités au XIII ${ }^{\mathrm{e}} \mathrm{s}$. Même les tenants de l'art de guérir étaient des moines et si la tradition pharmaceutique ne s'est pas éteinte pendant cette période, c'est parce qu'elle trouva asile dans les couvents, où le religieux préposé à la pharmacie s'appelait couramment apotecarius dès la fin du $\mathrm{x}^{\mathrm{e}} \mathrm{s}$. Pendant plusieurs siècles, pratiquement 
du $\mathrm{VI}^{\mathrm{e}}$ au XII ${ }^{\mathrm{e}}$, l'art de guérir était ainsi entre les mains du clergé et les quelques écrits pharmacologiques de l'époque sont l'œuvre d'ecclésiastiques: au bénédictin allemand Raban Maur (Rabanus, 776-856), nous devons les trois volumes de la Physica consacrés aux maladies et aux médicaments; à l'évêque de Rennes Marbode (10351113), le lapidaire De gemmis qui décrit les vertus médicinales de soixante pierres nobles; à la mystique allemande Hildegard von Bingen (1098-1179), le Liber simplicis medicinae et le Liber compositae medicinae; etc.

$\mathrm{Au}$ monastère de Monte Cassino, fondé par saint Benoît en 529, les moines médecins pouvaient puiser dans les collections d'écrits hippocratiques et galéniques conservés grâce au zèle des copistes byzantins et des traducteurs syriens et arabes. En ce qui concerne la pharmacie, ils se contentèrent au début de traduire en latin des formulaires de prescriptions. À la même époque, l'homme d'État et écrivain romain Aurelius Cassiodorus (480-575) se retirait de la vie publique, entrait dans l'ordre des bénédictins et installait en 540, dans sa propriété de Vivarium, une sorte d'académie monastique qu'il dota d'une riche bibliothèque. Dans son De institutione divinarum, il incita les moines à traduire les meilleurs ouvrages de la littérature médico-pharmaceutique grecque. Ainsi naquit une première école de traduction en Occident. Ce n'est toutefois qu'au $\mathrm{XI}^{\mathrm{e}}$ s., avec l'arrivée en Italie de Constantin l'Africain (1015-1087), que la traduction prit son véritable essor. Arrivé à Salerne en 1065, après avoir acquis un solide fonds scientifique au cours de voyages en Orient, ce Carthaginois se convertit au christianisme en 1070 et entra au Mont-Cassin, où il passa le reste de sa vie à traduire. Ses traductions latines des œuvres d'Hippocrate et de Galien à partir des versions arabes restaurèrent l'étude de la science médicale grecque en Europe. Auteur lui-même d'un Glosario de hierbas y farmacos, il mit aussi en latin le Kitab al-Itimad (De la fiabilité des drogues simples) d'Ibn al-Djazzar (?-1004) sous le titre de Liber de gradibus et, en collaboration avec son élève Johannes Afflacius ou Saracenus, le Kitab al-Malaki d'Haly Abbas sous le titre de Liber Pantegni (Tout l'art).

Avant de se retirer du monde, Constantin avait enseigné à l'École de Salerne, créée $\mathrm{au} \mathrm{IX}^{\mathrm{e}} \mathrm{s}$., où les diverses disciplines médico-pharmaceutiques étaient exposées en grec, en latin, en arabe et en italien. Elle attirait des savants de toute l'Europe chrétienne et de l'Espagne juive et musulmane. La pharmacie y était déjà indépendante de la médecine vers 1076 et sa renommée repose en partie sur un traité de vulgarisation rédigé en dialecte napolitain auquel on a donné le nom de Regimen sanitatis Salernitatum ( $\mathrm{xI}^{\mathrm{e}} \mathrm{s}$.). Jean de Milan, médecin salernitain, le mit en vers latins pour Robert, duc de Normandie, sous le titre de De conservando valetudine (v. 1100). Ce recueil de recettes se développa au fil des temps pour devenir l'Antidotarium Nicolai, lequel ne doit pas être confondu avec le Dynameron ( $\mathrm{xIII}^{\mathrm{e}} \mathrm{s}$.), l'antidotaire de Nicolas Myrepsos, qui comprenait plus de deux mille cinq cents remèdes grecs, romains, arabes, juifs et chrétiens.

Les médecins juifs qui participèrent à la fondation de Salerne furent aussi parmi les premiers maîtres des universités qui se créèrent à Bologne, à Montpellier, à Salamanque et ailleurs, dont l'enseignement put bénéficier de la masse de connaissances libérée par les travaux du Colegio de Traductores de Tolède. Dernier foyer de la culture arabe en Europe, Tolède avait été reconquise en 1085 par Alphonse IV de Castille et les richesses de ses bibliothèques attirèrent les érudits des quatre coins de l'Europe. En 1135, l'archevêque Raymond y fonda un collège de traducteurs où, pendant plus d'un siècle et demi, des Italiens, des Français, des Anglais, des Juifs, des Flamands 
s'illustrèrent aux côtés des Espagnols dans un gigantesque projet de traduction. Le domaine médico-pharmaceutique eut la bonne fortune de trouver un interprète de qualité en la personne de Gérard de Crémone (1114-1187) qui, arrivé à Tolède en 1167, apprit l'arabe et consacra les vingt dernières années de sa vie à la traduction en langue latine. Dans son immense production (quelque soixante-quinze titres), la pharmacie est représentée par le Canon d'Avicenne, avec son deuxième livre sur les drogues simples et le cinquième sur les médicaments composés, l'Aqrabadin al-kabir, formulaire de médicaments composés de Rhazès, le Kitab al-Adwiya du médecin et vizir de Tolède Ibn Wafid al-Lahmi (999-1068) qu'il intitula De medicamentis simplicibus, le Kitab at-Tasrif du médecin cordouan Abu al-Qasim az-Zahrawi (?-1009) dont les livres vingt-sept et vingt-huit traitent des drogues simples et de leur préparation.

L'expérience collective de Tolède ne doit pas éclipser l'effort de nombreux traducteurs qui œuvraient isolément. Stéphane d'Antioche (dit Étienne le Philosophe, $\mathrm{XII}^{\mathrm{e}}$ s.), originaire de Pise, latinisa le Kitab al-Malaki d'Haly Abbas sous le titre de Liber regius (ou Regalis disposition, 1127) en le complétant d'un glossaire grec-latinarabe Medicamentorum omnium breviarium. Abraham et Simon de Gênes traduisirent le vingt-huitième livre du Kitab at-Tasrif, le baptisant Liber servitoris. Le médecin et astronome Gérard de Sabbionetta ( $\mathrm{XIII}^{\mathrm{e}} \mathrm{s}$.) s'est rendu célèbre par ses traductions en italien de Galien. Stéphane de Saragosse retraduisit le Kitab al-Itima d'Ibn al-Djazzar en le baptisant Adminiculum ou Liber fiduciae de simplicibus medicinis (1233). Le dominicain allemand Albertus Magnus (1193-1280), auteur lui-même d'une encyclopédie sur les vertus thérapeutiques des plantes (Summa naturalium), fournit une nouvelle version latine du Canon d'Avicenne. En 1271, Moïse Faradj Ibn Salim (latinisé Faragut), médecin juif au service de Charles d'Anjou, roi de Naples, traduisit le Kitab al-Hawi de Rhazès qui, sous le titre de Liber continens, connut une extraordinaire diffusion; une édition de 1486 y joignit un glossaire arabe-latin des noms de drogues. En 1290, Abraham Tortuosiensis et Simon Januensis intitulèrent Liber de simplicibus medicinis leur traduction de l'arabe d'un ouvrage de Sérapion (Ibn Sararbiyun, $\mathrm{IX}^{\mathrm{e}}$ s.). Le XIII ${ }^{\mathrm{e}}$ s. vit aussi paraître des versions en catalan (Libre de les medicines particulars) et en hébreu du Kitab al-Adwiya d'Ibn Wafid. Au tournant du siècle, Blaise Ermengaud (dit Blaise de Montpellier), médecin de Philippe le Bel, signa des traductions latines du Kitab as-Sumum (Livre des poisons) de Maïmonide et du Maqala fi t-Tiryaq (Traité de la thériaque) d'Averroès. À l'autre bout de l'Europe, le médecin turc Ishaq Ibn Murad ( $x v^{\mathrm{e}} \mathrm{s}$.), auteur d'un livre sur les drogues simples (1390), mit dans sa langue le Kitab Taqwim (Almanach de santé) d’Isa Ibn Djazla, médecin chrétien de Bagdad converti à 1'Islam.

La fin du Moyen Âge vit poindre les premières traductions en langue vulgaire. Outre la catalane déjà citée, en Allemagne, Hildegard von Bingen avait dicté en latin ses deux livres sur les remèdes simples et composés à deux moines chargés de les mettre en allemand (v. 1155); en France, le clerc augustin Jean Sauvage (xIv ${ }^{\mathrm{e}}$ s.) traduisit le Thesaurus pauperum, recueil de recettes thérapeutiques attribué à Pietro di Giuliano (dit Petrus Hispanus), médecin de Grégoire X, qui devint en français Le Tresor des povres. Par ailleurs, des traductions en arabe, comme celle de l'Antidotarium Nicolai avant même la moitié du $\mathrm{XIV}^{\mathrm{e}} \mathrm{s}$., montrent que l'art médico-pharmaceutique arabe, après avoir nourri l'Occident médiéval pendant plus de trois cents ans, venait à son tour s'abreuver aux sources du savoir occidental. Ses jours, cependant, étaient 
comptés et il devait bientôt succomber aux forces humanistes qui, à partir de 1400, allaient marquer profondément tous les courants de pensée en Europe.

\section{Le renouveau des esprits}

Parti d'Italie, le mouvement humaniste gagna très vite toute l'Europe. La prise de Constantinople par les Turcs, en 1453, ne fit que l'accélérer et l'amplifier avec l'arrivée d'une foule de savants chassés des derniers bastions de l'hellénisme. La médecine humaniste devint universelle grâce aux liens scientifiques et culturels tissés entre les nations par les universités, grâce aux grandes découvertes révélatrices de remèdes (gaïac, mecheocan, etc.) venus du Nouveau Monde et grâce à l'invention de l'imprimerie qui favorisa la diffusion des écrits médico-pharmaceutiques. Dominée par quelques grands noms - l'Italien Fracastoro, le Flamand Vésale, le Français Paré et, pour la pharmacie, le Suisse Paracelse - elle remit en question les acquis de la science et s'employa à se dégager de l'emprise des Arabes. Paracelse (1493-1541), qui était médecin et alchimiste, opposa sa théorie chimique - l'homme est un composé chimique et doit donc être soigné par des composés chimiques — à la doctrine galénique. Il expérimenta les vertus de toutes les plantes pour découvrir la cause de leurs effets pharmacodynamiques; il introduisit dans la thérapeutique des remèdes tirés du règne minéral et généralisa l'emploi de l'antimoine, dont les propriétés avaient été observées pour la première fois au $\mathrm{xv}^{\mathrm{e}} \mathrm{s}$. par le bénédictin allemand Basile Valentin, médecin et chimiste, et exposées dans son livre Triumphwagen der Antimonii, traduit en latin sous le titre de Currus triumphalis antimonii. À partir du xvi ${ }^{e}$ s., la pharmacologie fut influencée par le mouvement chimique et l'effort porta sur la mise au point de traitements médicamenteux plus scientifiques. C'est alors que la thérapeutique de la syphilis abandonna les cures purgatives classiques pour les remplacer par le bois de gaïac, préconisé par le chevalier allemand Ulrich von Hütten (1488-1523) dans son opuscule célèbre De guaiaci medicina et morbo gallico (1519), puis par les cures mercurielles. En France, c'est le botaniste et maître apothicaire Nicolas Houel (1520-1584), auteur d'un Traité de la thériaque et du mithridate (1573), qui dirigea la pharmacologie dans la voie scientifique en créant à Paris, en 1576, un véritable enseignement de l'«art d'apothicairerie».

Malgré les attaques dont ils étaient la cible des Paracelse et autres Vésale, les auteurs grecs, latins et arabes continuèrent à attirer les traducteurs, parmi lesquels beaucoup usaient encore du latin resté la langue de prédilection des lettrés et des savants de la Renaissance. En France, le médecin Jean de Gorris traduisit l'Alexipharmaca de Nicandre. Aux Pays-Bas, Johannes Guinterus (Jan Winter) signa une version du De re medica de Paul d'Égine qui fut révisée par le médecin et botaniste malinois Rembert Dodoens (dit Dodonée, 1517-1585) et publiée sous le titre de Paulus Aeginata a Joanne Guintero latine conversus, a Remberto Dodonaeo ad graecum textum accurate collatus et recensitus (1546). Une traduction latine des ouvres de Paul d'Égine fut par ailleurs insérée par Henri Estienne dans sa collection Artis medicae principes (1567). Un autre traité de matière médicale très en faveur auprès des traducteurs fut celui de Dioscoride. Ermolao Barbaro, patriarche de Venise et humaniste de renom, en donna la première traduction latine qui, bien que faite entre 1481 et 1489, ne vit le jour qu'en 1516. La même année, Jean Ruelle (1471-1537), professeur à la Faculté de médecine de Paris, publiait sa version Pedacii Dioscoridis 
Anazarbei de medicinali materia libri quinque. Deux ans plus tard, paraissait une nouvelle traduction du médecin florentin Marcello Adriani (1464-1521) intitulée Pedacii Dioscoridae de medica materia libri sex (1518), à laquelle succédèrent celle du médecin de Padoue Pietro-Andrea Mattioli (1501-1577) en 1554, celle de l'Allemand Johannes Haynpol (dit Janus Cornarius) intitulée Pedacii Dioscoridae Anazarbensis de materia medica libri V (1557) et, en 1598, celle de Jean-Antoine Sarrazin (15471598), médecin à Montpellier, Pedacii Dioscoridis Anazarbei opera quae extant omnia. Le médecin et orientaliste italien Andrea Alpago (?-1520), qui travailla longtemps au consulat de Venise à Damas, non seulement révisa la version du Canon d'Avicenne réalisée par Gérard de Crémone mais, en outre, signa la traduction De limonibus d'un traité d'Ibn al-Baitar.

Des traductions latines se faisaient même à partir de langues vulgaires. Le médecin et botaniste français Charles de l'Escluse (dit Clusius, 1526-1609), remarquable polyglotte qui maniait avec aisance le latin, le grec, le flamand, l'anglais, l'allemand, l'italien, l'espagnol et le portugais, avait à son actif de nombreuses traductions de ces trois dernières langues. Ainsi, de l'italien, l'Antidotarium, sive de exacta componendorum miscendorumque medicamentorum ratione libri tres (1561) est traduit d'une pharmacopée florentine, le Ricettario. Du portugais, la version qu'il donna des Coloquios dos simples de Garcia de Orta sous le titre d'Aromatum et simplicium aliquot medicamentorum apud Indios nascentium Historia (1567) fut le point de départ de multiples traductions en italien et en français entre 1576 et 1619. De l'espagnol, il traduisit l'Historia medicinal de las cosas que se traen de nuestras Indias occidentales, que sirven en medicina de Nicolas Monardès, qui devint De simplicibus medicamentis ex Occidentali India delatis, quorum in medicina usus est (1574), et le Tractado de las drogas y medicinas de Christoforo Acosta, qui devint Aromatum et medicamentorum in Orientali India nascentium liber (1582). Comme pour témoigner du caractère universel de l'art médico-pharmaceutique de la Renaissance, il se trouva même un clerc mexicain, Juan Badiano, pour mettre en latin la matière médicale d'un codex indien sous le titre Libellus de medicinalibus Indorum herbis (1552).

L'imprimerie, si elle favorisa la diffusion des connaissances, engendra par ailleurs une nouvelle classe de lecteurs qui n'entendaient plus le latin. Il s'ensuivit une demande accrue de traductions en langue vulgaire. En France, les livres pharmacologiques de Galien trouvèrent ainsi des interprètes en Jean Canappe, médecin de François I ${ }^{\text {er }}$, pour Deux livres des simples (V et IX) (1542), en Jean Bauhin (1511-1582), médecin protestant réfugié à Bâle, pour Des simples, livre V (1544), en Martin Grégoire pour Les trois premiers livres sur la composition des médicaments en général (1545), en Jean Brêche (1514-1553), jurisconsulte, pour un Épitomé ou Abrégé des trois premiers livres De la Composition des médicaments (1545), en Hervé Fayard, médecin, pour Galen. Sur la faculté des simples médicaments (1548). L'humaniste protestant Michel Cop traduisit sur le latin la Pharmacia simplicia (1532) de Paul d'Égine et le bénédictin François Mathée fit de même pour Les six livres de Pedacion Dioscoride d'Anazarbe de la matière médicale (1559), mis pour la première fois en français; dix ans plus tard, une nouvelle version d'un anonyme parut à Paris sous le titre Les propriétés des simples, contenues en six livres de Dioscoride, rapportées aux accidents qui peuvent advenir à chaque partie du corps (1569). Le médecin et poète Jacques Grévin (1538-1570) mit en vers français Les Euvres de Nicandre (1567). Antoine Du Pinet (v. 1510-1566) publia l'Histoire naturelle de Pline traduite en français (1564). Sous la plume de Lucas 
Tremblay, le De virtutibus (vel viribus) herbarum, faussement attribué au poète Aemilius Macer (70-16 av. J.-C.) et publié à Naples en 1477, devint Les Fleurs du livre des vertus des herbes (1588). Une traduction anonyme de Mésué l'Ancien, réalisée non à partir de l'arabe mais sur le Commentarium J. Tagautii de purgantibus medicamentis simplicibus libri II de Jean Tagaut (?-1546), doyen de la Faculté de médecine de Paris, parut sous le titre Canons universels de Jean Mésué des simples médicaments purgeants.

Parallèlement, se multipliaient les traductions d'ouvrages néo-latins. Après une première version anonyme intitulée Le Régime très utile et très profitable pour conserver et garder la santé du corps humain (v. 1495), le Regimen sanitatis de Salerne fut retraduit en vers par l'avocat Geoffroy Le Tellier, qui en fit le Retardement de la mort par bon régime ou conservation de santé, jadis envoyé par l'école de Salerne au roi d'Angleterre (1561). L'ouvrage Hortus sanitatis du médecin et naturaliste allemand Joannes de Cuba $\left(\mathrm{xv}^{\mathrm{e}} \mathrm{s}\right.$.) parut à Paris dans une traduction anonyme, Le Jardin de Santé (1501). Parmi les ouvrages néo-latins français, les Formulae remediorum quibus vulgo medici utuntur (1560) de Pierre des Gorris (?-1560), médecin à Bourges, devinrent Les Formes et remèdes desquels les médecins usent en toutes maladies (1570) sous la plume de Jean Rivière; en 1574, la Pharmacopoea du médecin humaniste Jacques Dubois (dit Sylvius), professeur au Collège royal, fut mise en français par André Caillé (v. 1515-1580), médecin-apothicaire à Lyon, qui mit aussi à son actif Le Jardin médicinal enrichi de plusieurs et divers remèdes et secrets (1577) traduit de l'Alexikepus, seu auxiliaris hortus (1575) du médecin Antoine Mizaud; la Pharmacopoea (1577) de Laurent Joubert, médecin du roi Henri II et chancelier de l'université de Montpellier, fut traduite par Jean-Paul Zangmaistre sous le titre La Pharmacopée de M. L. Joubert, ensemble les annotations de J. P. Zangmaisterus (1581).

Parmi les œuvres néo-latines étrangères, une place de choix revint à l'Italie où, au $\mathrm{XVI}^{\mathrm{e}}$ s., Padoue représentait le centre de la science médico-pharmaceutique. Les célèbres Commentarii in libros sex $P$. Dioscoridis de materia medica du médecin siennois Pietro Andrea Mattioli connurent plusieurs interprètes, le premier n'étant autre que Du Pinet, avec ses Commentaires de P. A. Matthiolus médecin Senois sur les six livres de Pedacius Dioscorides Anazarbéen de la matière médicale (1561); il fut suivi des Commentaires de M. Pierre André Matthiole médecin senois sur les six livres de Ped. Dioscoride Anazarbéen de la matière médicinale [...] mis en français sur la dernière édition latine par le médecin et botaniste Jean des Moulins (1530-1622) et d'une version partielle par Geoffroy Linocier dans son Histoire des plantes (selon Fuchs, Matthiolus, Gesner et al.) (1584). Le médecin Pierre Tolet, condisciple de Rabelais, traduisit l'ouvrage De radice purgante quam Mechioacan vocant (1569) de son confrère italien Marcello Donati sous le titre Traité de l'admirable vertu et accomplissement des facultés pour la santé et la conservation du corps humain de la racine nouvelle de l'Inde Mechiaacan proprement nommée Rhaindice (1572). Lazare Boet mit en français le De Venenis eorumque remediis liber (Traité des venins, 1593) de Pierre d'Abano, médecin et alchimiste de Padoue, et Isaac Constans (1564-1630), professeur de médecine à l'Académie protestante de Montauban, fit de même pour l'Antidote des maladies pestilentielles (1595) de l'humaniste Marsilio Ficino. Le chirurgien Joseph Du Chesne (dit Quercetanus, 1545-1609) traduisit d'un anonyme néo-latin le Traité de la cure générale et particulière des arcbusades. Avec l'antidotaire spagirique pour préparer et composer les médicaments (1576). 
Les traducteurs s'intéressèrent aussi à plusieurs auteurs néo-latins allemands. C'est Jean Chéradame (?-1543), médecin à Paris, qui se chargea de l'opuscule sur le gaïac d'Ulrich von Hütten, dont il fit L'Expérience et approbation [...] touchant la médecine du bois dit guaiacum (1520) un an seulement après la publication de l'original. Les ouvrages du médecin et botaniste Leonhart Fuchs, professeur à l'Université de Tübingen, tentèrent plusieurs traducteurs: son Historia stirpium (1542), qui décrit une centaine de plantes médicinales, passa par les mains de Guillaume Guéroult (1507-1564) en 1548 (Histoire des plantes, mise en commentaire par Léonard Fuchs, médecin), d'Hervé Fayard la même année (partielle, jointe à sa traduction de Galien), d'Éloi de Maignan en 1549 (Commentaires très excellents de l'histoire des plantes composés premièrement en latin par L. Fousch, traduits en langue française par un homme savant en la matière), de Jean Belon en 1549 encore (Histoire des plantes de M. L. Fuchs, avec les noms grecs, latins et français) et de Geoffroy Linocier (?-1620) en 1584. Deux versions du Dispensatorium, sive pharmacorum conficiendorum ratio du botaniste Valerius Eberwein (dit Cordus) parurent à quelques années d'intervalle, l'une appelée Le Guidon des apoticaires, c'est-à-dire la vraie forme et manière de composer les médicaments (1572) par Pierre Coudemberg, médecin-apothicaire à Anvers et créateur du jardin botanique de cette ville en 1548 (celui de Paris ne fut créé qu'en 1576 par Nicolas Houel), l'autre par André Caillé, déjà cité, également intitulée Le Guidon des apoticaires en 1578. De Suisse vinrent des œuvres de Theophrastus von Hohenheim (dit Paracelse) et de Conrad von Gesner (dit Evonymus Philiatros). De ce dernier, le Thesaurus de remediis secretis (1552) fut traduit, pour la première partie, par l'érudit Barthélémy Aneau (?-1561) sous le titre Trésor de Evonyme Philiatre des remèdes secrets (1555), pour la seconde par le docteur-régent de la Faculté de médecine de Paris Jean Liébault (1534-1596), sous le titre Quatre livres des secrets et de la philosophie chimique (1573); une traduction partielle parut encore dans l'Histoire des plantes (1584) de Linocier. Paracelse trouva des interprètes en Jean Boiron, marchand lyonnais, pour son Traité du vitriol (1581) et en Lazare Boet pour son Traité des vertus et propriétés merveilleuses des vers, serpens, araignes, crapaux et cancres (1593).

Dans le même temps, d'autres traducteurs se penchaient sur des originaux écrits en langue vulgaire. Du néerlandais, Charles de l'Escluse traduisit le Cruydeboeck (1554) du médecin et botaniste flamand Rembert Dodoens sous le titre d'Histoire des plantes, en laquelle est contenue la description entière des herbes [...] qui viennent en usage en médecine (1557); du portugais, il donna l'Histoire des drogues, espiceries et certains médicaments simples qui naissent ès Indes et en Amérique, traduite des Coloquios dos simples de Garcia de Orta, qu'il avait précédemment déjà mis en latin. Le Kreutter Buch du médecin et botaniste allemand Jérome Bock devint le Nouvel herbier des plantes qui croissent en Alemagne dès 1559. Du médecin et botaniste espagnol Nicolas Monardes parurent l'Histoire des simples médicaments nouvellement apportés des terres neuves et, par les soins de l'érudit parisien Jacques Gohorry (15201576), le Brief traité de la racine de mechoucan venue d'Espagne nouvelle (1572). De l'italien, Claude Rocard transposa Les Caprices de M. Léonard Fioravanti touchant la médecine, qui sont plusieurs et diverses médecines (1586) du médecin bolognais Leonardo Fioravanti, l'inventeur du baume contre les rhumatismes qui porte son nom.

Une activité de traduction similaire existait dans les autres pays. En Italie, la matière médicale de Dioscoride fut traduite pour la première fois sous le titre de 
Dioscoride fatto di greco in italiano (1542) par da Longiano, de son vrai nom Fausto Sebastiano (1502-?) et retraduit ensuite par Mattioli sous le titre de Di Pedacio Dioscoride Anazarbeo Libri Cinque. Della historia et materia medicinale tradotti in lingua volgare (1544), puis encore par Marcantonio Montigiani, médecin à San Gimignano, qui l'appela Dioscoride Anazarbeo della materia medicinale tradotto in lingua florentina (1547). En Espagne, c'est Andrés Laguna (1499-1560), médecin du pape Jules III, qui se chargea de la première traduction de Pedacio Dioscorides Anazarbeo. Acerca de la materia medicinal. Traducido de lengua griega en la vulgar castellana (1555) et le médecin et philosophe Juan Jarava publia une Historia de las yervas y plantas (1557) traduite de l'Historia stirpium de Leonhart Fuchs. En Grande-Bretagne, Thomas Paynell traduisit en 1533 le De guaiaci medicina d'Ulrich von Hütten; dans son Castel of Health (1539), le médecin et diplomate Thomas Elyot (1490-1546) inséra une traduction des prescriptions et remèdes de Galien et d'autres médecins de l'Antiquité; en 1577, John Frampton fit paraître Joyfull Newes out of the Newe Founde Worlde, traduit de la Historia medicinal de las cosas que se traen de nuestras Indias occidentales de Nicolas Monardés; Henry Lyte se servit de la version française de l'Escluse pour mettre en anglais le Cruydeboeck de Dodonée, qu'il intitula A Niewe Herball or Historie of Plantes by the learned R. Rembert Dodoens and nowe first translated out of French into English (1578). En Allemagne, l'humaniste Thomas Murner (1475-1537) traduisit le livre de son compatriote Ulrich von Hütten en l'intitulant Von der wunderbaren Arznei des Holz Guaiacum genannt; dès 1532-1537, l'Herbarum vivae eicones (1530-1536) du médecin Otto Brunfels fut mis en allemand sous le titre Contrafeyt Kreüterbuch in Teutscher Sprach; l'Historia stirpium (1542) de Fuchs connut le même sort dès 1543 et la première version allemande de la Materia medica de Dioscoride parut en 1546. Aux Pays-Bas, une traduction flamande, la toute première de Dioscoride dans une langue vulgaire, avait vu le jour en 1520.

Du point de vue linguistique, la Renaissance dut composer entre son désir de cultiver les langues anciennes et la nécessité d'utiliser les langues vulgaires voulue par la naissance des nationalismes. Cette confrontation permanente a engendré les premières entreprises lexicographiques, y compris dans le domaine pharmaceutique. Andrea Alpaga annexa un glossaire arabe-latin à sa traduction du Canon d'Avicenne; Charles de l'Escluse, dans sa version de l'Histoire des plantes, ajouta le français au lexique latin-néerlandais original du Cruydeboeck de Dodonée; en Allemagne, Lorenz Fries (dit Phrisius) publia des Synonyma und gerecht Usslegung der Wörter so man den in der Artzny - allen Krütern, Wurzlen, Blummen, Somen, Gesteinen, Safften und anderen Dingen - zum schreiben ist (1519), latin-hébreu-arabe-grec et «mancherlei Tütschen Zungen» (toutes sortes de dialectes allemands).

\section{Le triomphe des Modernes}

À l'admiration outrancière de la Renaissance pour les Anciens succédèrent des siècles de réaction qui marquèrent la religion, la politique et la science. L'Antiquité n'intéresserait plus que dans la mesure où elle pouvait servir les aspirations nationalistes. Des nations devinrent protestantes de religion, républicaines de gouvernement. La science devint rationaliste et des académies scientifiques firent leur apparition un peu partout. Partout se développa l'idée de progrès, le mépris de la tradition, la confiance de l'individu en soi-même. Les Modernes sortirent vainqueurs du XvII ${ }^{\mathrm{e}}$ siècle. 
Pour la pharmacie, ce $\mathrm{XVII}^{\mathrm{e}}$ s. constitua une étape de transition. Embarrassée encore par de nombreuses données empiriques, elle demeura stationnaire pendant toute la période, même si la thérapeutique alla puiser dans la science chimique des éléments nouveaux. Le médecin et chimiste belge Jean-Baptiste Van Helmont, disciple de Paracelse, fut le premier à utiliser le terme «chimique» dans son ouvrage Ortus medicinae (1648). Les partisans du latin étaient encore nombreux parmi les érudits et les savants. C'est d'ailleurs dans cette langue que parut la première revue médicale, Miscellanea Curiosa Medico-Physica (Allemagne, 1670).

Même la traduction médico-pharmaceutique se servait toujours du latin. C'est ainsi que Fortun Plemp (dit Plempius), à Louvain, intitula sa version de la partie du Canon d'Avicenne relative aux drogues simples Clarissima [...] Ibn Tsina [...] canon medicinae, interprete et scholiaste Vopisco Fortunato Plempio (1658); c'est en latin aussi que l'orientaliste français Antoine Galland (1646-1715) traduisit le Kitab alDjami, traité de pharmacognosie d'Ibn al-Baitar. À l'inverse, des œuvres néo-latines étaient mises en langue vulgaire, telle l'Historia generalis plantarum du médecin lyonnais Jacques Dalechamps, traduite en français par l'interprète de Dioscoride Jean des Moulins; tel encore le Serment des apothicaires (1608) qu'aurait composé en vers latins Jean de Renou, médecin du roi Henri III, et que mit en français le médecin lyonnais Louis de Serres en 1624.

S'il se trouvait encore des hellénistes pour traduire la matière médicale de Dioscoride en anglais - car, étrangement, le traité ne fut pas mis dans cette langue avant le milieu du XVII $\mathrm{e}$. — c'est toutefois vers les langues latines que les traducteurs se tournèrent de plus en plus, tel Antoine Colin qui intitula Traité des drogues et des médicaments qui naissent aux Indes (1619) la version qu'il donna du Tratado de las drogas y medicinas de las Indias Orientales, con sus plantas (1578) du médecin et naturaliste Cristofora Costa.

Dans un $\mathrm{XVIII}^{\mathrm{e}} \mathrm{s}$. où la vie intellectuelle était régentée par les philosophes, où la philosophie dominante était celle de la raison, où seule la marche du progrès basé sur la science importait, un revirement complet s'effectua dans les usages médicopharmaceutiques. Linné (Fundamenta botanica) jetait les bases du système de classification des plantes; Lavoisier, Priestley et Scheele établissaient la nomenclature chimique; en Allemagne, Samuel Hahnemann inventait 1'homéopathie. Les conditions indispensables au véritable progrès scientifique étant réunies, l'art médicopharmaceutique se libéra définitivement du dogmatisme galénique, de l'alchimie, de l'empirisme fantaisiste qui avait encore maintenu l'emploi thérapeutique des pierres tout au long du siècle précédent. Les traducteurs commencèrent à se pencher sur des ouvrages contemporains, tels Hahnemann (1755 -1843), qui mit en allemand la Pharmacology de Monroe et la Materia medica de W. MacCullen. Cela n'empêcha pas l'orientaliste Abraham Anquetil-Duperron (1731-1805) de s'intéresser au Zend Avesta, dont il avait retrouvé un exemplaire complet en 1758, et d'en donner la première version complète établie sur 1'original zend en 1771. Une autre découverte importante pour l'Occident fut le Pen-ts'ao kang mou (Traité de matière médicale) de Li Che-tchen (1518-1593), médecin sous les Ming, dont une traduction partielle fut publiée en 1731 par le jésuite français Jean-Baptiste du Halde (1674-1743).

Amorcée à la Renaissance, la compilation de dictionnaires s'est poursuivie et la pharmacognosie se retrouve dans des ouvrages comme le Dictionnaire des plantes médicinales (1783-1789) latin-russe-grec-français-allemand-anglais du Russe N. 
Maksimovitch ou 1'Allgemeines Polyglotten-Lexikon der Naturgeschichte (1793-1798) allemand-néerlandais-danois-suédois-anglais-français-italien-espagnol-portugais de 1'Allemand P. Nemnich.

\section{Au seuil des temps nouveaux}

La pharmacologie du XIX ${ }^{e}$ s., entièrement bouleversée par les acquisitions incessantes des sciences chimiques, vit s'accomplir en quelques années un véritable miracle dans le domaine du médicament. Des pharmaciens - Davy, Klaproth, Berzelius — n'hésitèrent pas à contribuer à la construction de l'édifice chimique. Les recherches analytiques et l'étude des constituants chimiques des plantes se développèrent rapidement. La chimie organique prit son essor vers 1830, entrainant le remplacement de la plupart des drogues végétales par leurs principes actifs et la création de composés organiques doués de propriétés médicamenteuses. L'importance prise par la pharmacie put se mesurer à l'organisation des premiers congrès internationaux: Brunswick en 1865, Paris en 1867, Vienne en 1869, Saint-Pétersbourg en 1874, Londres en 1881, Bruxelles en 1885. En France, la dénomination d'apothicaire, devenue péjorative, fut abandonnée en faveur de celle de pharmacien.

L'avènement de la chimie pharmaceutique n'effaça toutefois pas l'intérêt pour les pharmacologies d'antan, que des découvertes récentes virent d'ailleurs raviver. Ainsi le papyrus Ebers, grand classique de la pharmacologie égyptienne, révélé en 1873 par l'égyptologue allemand Georg Ebers et édité par lui en 1875, fut-il traduit par Joachim sous le titre de Papyros Ebers. Das älteste Buch über Heilkunde (1890). Le Zend Avesta, découvert au siècle précédent par Anquetil du Perron, fit l'objet de plusieurs versions nouvelles: en français par l'orientaliste Eugène Burnouf (1801-1852) sous le titre d'Avesta (1829-1833), par l'orientaliste belge Charles de Harlez (18321899) sous le titre de Avesta (1875-1878) et par l'orientaliste James Darmesteter (1849-1894) sous le titre de Le Zend Avesta, traduction nouvelle avec commentaire historique et philologique (1892-1893); en allemand, par l'orientaliste Friedrich von Spiegel (1820-1905), d'après l'ancienne version pehlvi de l'époque sassanide, sous le titre de Zend Avesta (1852-1863) et par l'indianiste Karl Geldner (1852-1929) sous le titre d'Avesta. Die heiligen Bücher der Parsen (1886-1895); en anglais, par Geldner encore, sous le titre d'Avesta. The Sacred Books of the Parsi (1886-1896). Le manuscrit de médecine indienne (v. 400) découvert à Koutcha, dans le Turkestan chinois par le lieutenant britannique A. Bower et contenant plusieurs chapitres de recettes diverses (antiseptiques, aphrodisiaques, pommades oculaires, etc.) fut mis en allemand par l'orientaliste August Hoernle (1841-1918). Le Pen-ts'ao kang mou, venu à la connaissance de l'Occident au XviII ${ }^{\mathrm{e}}$ s., trouva de nouveaux interprètes en A. J. C. Geerts (1843-1883) - traduction partielle pour son ouvrage Les produits de la nature chinoise et japonaise (1878-1883) - en P. Dabry de Thiersant - traduction de la matière médicale végétale pour sa Médecine chez les chinois (1863) - et en F. de Mély (18511935) - traduction de la matière médicale minérale dans son Lapidaire chinois (1898) — pour le français, ainsi qu'en F. Porter Smith et E. Bretschneider pour une version partielle anglaise (1894). En 1873, le botaniste français Paul Savatier traduisit les livres Kwa-wi de la botanique japonaise avec l'aide de M. Saba.

Parmi les ouvrages de l'Antiquité gréco-romaine, le Corpus hippocraticum connut une nouvelle jeunesse sous la plume du médecin et philologue Émile Littré (1801- 
1881), qui publia ses Euvres complètes d'Hippocrate de 1839 à 1853; en GrandeBretagne, F. Adams retraduisit The Seven Books of Paulus Aeginata (1844-1847); le De re medica de Celse subit le même sort des mains du médecin français Félix Savart (1791-1841).

La pharmacologie persane et arabe également attirait toujours les traducteurs. Le Chah-name du poète persan Firdousi (940-1020) — influencé par l'Avesta et dans lequel le «parmacologue» est, parmi les médecins, «celui qui soigne par les plantes» - fut mis en français (Le livre des rois, 1838-1855) par l'orientaliste allemand naturalisé Julius von Mohl (1800-1876) et en allemand (Firdosi's Königsbuch (Schahname), 1890) par l'orientaliste Friedrich Rückert (1788-1866). Le Kitab al-Abniya (v. 970) d'Abu Mansur Muwaffaq, qui compte parmi les documents les plus anciens en néo-perse, devint en allemand Die pharmakologische Grundsätze des Abu Mansur Muwaffaq bin Ali Harawi (1893) par les soins d'A. C. Achundow. Joseph von Sontheimer traduisit Avicenne dans Zusammengesetzte Heilmittel der Araber. Nach dem 5. Buch des Canons von Ebn Sina (1845), après avoir donné déjà le Kitab al-Djami d'Ibn al-Baitar, qu'il intitula Grosse Zusammenstellung über die Kräfte der bekannten einfachen Heil- und Nahrungsmittel (1840-1842). Quelques années auparavant, ce même traité de pharmacognosie avait bénéficié d'une version partielle en latin de Friedrich Diez, Eleuchus materiae medicae Ibn Beitharis Malacensis (Leipzig, 1833). Il fut aussi mis en français sous le titre de Traité des simples d'Ibn el-Beithar (1877, 1881, 1883) par Lucien Leclerc, auquel on doit en outre le Traité de matière médicale arabe d'Abd er-Rezzaq l'Algérien (1874), médecin du xviII ${ }^{\mathrm{e}}$ s. Le Kitab as-Sumum de Maïmonide devint en français, traduit à travers la version hébraïque par Michel Rabbinowicz, le Traité des poisons (1865) et, en allemand, traduit également de l'hébreu par Moritz Steinschneider, Gifte und ihre Heilung, eine Abhandlung des Moses Maimonides auf Befehl des aegyptischen Wezirs verfasst (1873).

La lexicographie pharmaceutique s'étoffa davantage avec le Dictionnaire pour pharmacies et drogueries (1843) russe-allemand-latin de H. A. Jenken, le Dictionnaire botanique (pour botanistes, médecins et pharmaciens) (1878) latin-russe-allemandfrançais-anglais de N. Annenkov, le Dictionnaire des termes courants de pharmacie (1882) latin-russe-ukrainien-allemand-français de K. Kruze, le Lexicon synonymorum pharmaceuticorum (1888) latin-polonais-russe-allemand-français-anglais de W. Wiorogorski, etc.

\section{Le siècle d'or de la pharmacie?}

Certains spécialistes n'ont pas hésité à écrire que le $\mathrm{xx}^{\mathrm{e}} \mathrm{s}$. peut être considéré comme le siècle d'or de la pharmacie. Il a vu la synthèse chimique s'organiser et s'industrialiser, donnant naissance à quantité de médicaments synthétiques. Les théories nouvelles de la biochimie végétale ont suscité de nombreux travaux de phytochimie. La découverte des sécrétions internes a relancé l'intérêt pour la zoothérapie. La mécanisation a permis d'inonder le marché de formes pharmaceutiques nouvelles. On imagine aisément que, dans un tel climat de progrès, la littérature pharmaceutique a pris des proportions impressionnantes, entraînant à sa suite une activité de traduction non moins considérable, à un point tel qu'il serait vain, sans doute, de vouloir donner une idée de sa diversité autrement qu'à l'aide de quelques exemples, tels Éléments de matière médicale et de thérapeutique traduits au début du siècle du neurologiste 
allemand Hermann Nothnagel, The Vitamin Hunters (1962) traduit par H. F. Bernays de l'Allemand Albert Haller, Drugs Pollution (1974) traduit par O. Ordish du Hollandais W. T. Hausch, Pharmacology of Hormones (1975) traduit par M. Abileah de l'Allemand M. Tansk, etc.

Une autre bonne mesure est fournie par l'expansion de la lexicographie pharmaceutique, tant monolingue que multilingue, qui n'a cessé de s'amplifier jusqu'à nos jours: Dictionnaire des termes pharmaceutiques (1903) latin-russe-allemand-français d'A. G. Klinge, Dictionnaire botanique des termes pharmaceutiques (1909) latin-russepolonais-allemand-français-anglais d'A. Snapir, Poliglota vade-mecum de internacia farmacia (1911) espéranto-anglais-français-allemand-espagnol-italien-néerlandaisrusse-suédois de C. Rousseau, Vocabularium pharmaceuticum (1924) allemand-anglais-français-italien d'A. Graa, Pharmazeutisch-chemisches Fremdwörterbuch (1927) de H. Apel, Dictionnaire des plantes médicinales les plus actives et les plus usuelles et leurs applications thérapeutiques (1935) unilingue français de P. P. Botan, Medical Drugs Terminology (1947) chinois-latin-allemand-anglais-français-japonais de H. Hua, Pharmazeutische Terminologie (1947) unilingue allemand d'O. Zekert, Pharmazeutisches Wörterbuch (1950) unilingue allemand de C. Hunnius, Diccionario alemánespañol de terminología química, farmaceútico y bioquímica (1955) de J. Chabas-Lopez, A Dictionary of Practical Materia Medica (1955) unilingue anglais de J. H. Clarke, A Dictionary of Terms in Pharmacognosy (1955) unilingue anglais de G. M. Hocking, Drug Guide of Botanical Drugs and Spices Throughout the World (1959) anglaisfrançais-allemand d'E. F. Steinmetz, Dictionnaire en sept langues à l'usage du pharmacien pratiquant (1963) allemand-français-anglais-italien-espagnol-grec-russe d'E. Steinbichler, Apotheker-lexikon (1966) unilingue allemand de S. Hahnemann, Dictionary of Pharmaceutical Science and Techniques. 1. Pharmaceutical Technology (1968) 2. Materia Medica (1980) anglais-français-italien-espagnol-allemand-latin d'A. Sliosberg, etc.

On aura remarqué que plusieurs des ouvrages cités comportent encore le latin. Il en est un autre qui s'est même intéressé à la matière médicale égyptienne, le Wörterbuch der ägyptischen Drogennamen (1959) de H. von Deines et H. Grapow. C'est que les traducteurs, en dépit des multiples orientations nouvelles qui les sollicitent, restent malgré tout attirés par l'art pharmaceutique des civilisations antérieures. En Angleterre, P. Ebbell traduit The Papyrus Ebers (1937); en Allemagne, W. Wrezinski publie la traduction de deux recueils de remèdes et recettes, le premier du règne de Thoutmosis III (dit papyrus Hearst, découvert en 1899), le second du règne de Toutankhamon (dit papyrus de Londres), sous le titre de Der Londener medizinischer Papyrus und der Papyrus Hearst (1912). En 1905, l'indianiste américain Charles Lanman (1850-1941) met en anglais le chapitre sur les toniques (Hasayana) de l'Atharva-véda et K. Bhishagratna fait de même pour la Susruta-Samhitâ (19071916). Une nouvelle version allemande du Zend Avesta. Die heiligen Bücher der Parsen (1910) paraît sous la signature de F. Wolff. De même, le Chah-name de Firdousi trouve un nouvel interprète en H. Kanus-Credé, qui publie Firdausi. Das Königsbuch (1967).

Quelques Grecs et Romains connaissent la même fortune: la Theriaka et 1'Alexipharmaka de Nicandre sont mises en anglais par A. S. Gow et A. F. Scholfield; la matière médicale de Dioscoride devient en allemand Pedianos Dioskurides aus Anazarbos Arzneimittellehre in fünf Büchern (1902) par les soins de J. Berendes et, en 
anglais, The Greek Herball of Dioscorides (1934) par ceux de R. T. Gunther; le De re medica de Celse subit le même sort, une version allemande de T. Meyer-Steineg en 1912 et une anglaise de W. G. Spencer de 1935 à 1938; deux nouvelles versions françaises paraissent de l'Histoire naturelle de Pline l'Ancien, l'une du latiniste Jacques André (1910-), l'autre du latiniste Alfred Ernout (1879-1973) de 1947 à 1955.

La pharmacologie arabe n'est pas moins fréquentée. Les chapitres sur les drogues du Kitab Firdaus (Paradis de la sagesse) de Rabban at-Tabari (810-855) sont traduits par Alfred Siggel sous le titre Die indischen Bücher aus dem Paradies der Weisheit (1951). Siggel est aussi l'auteur d'une version du Kitab as-Sumum (Livre des poisons) de Gabir Ibn Haiyan intitulée Das Buch der Gifte des Gabir Ibn Hayyan. Arabischer Text in Faksimile, übersetzt und erläutert (1958). En Allemagne encore, Karl Garbers publie du Kitab Kimiya d'al-Kindi (?-870) la traduction Buch über die Chemie des Parfüms und die Destillationen von Yaqub ben Ishaq al-Kindi. Ein Beitrag zur Geschichte der arabischen Parfümchemie und Drogenkunde aus dem 9. Jahrhundert (1948). AlKindi est aussi traduit par l'arabisant anglais Martin Levey, qui en donne The Medical Formulary or Aqrabadhin of Al-Kindi, translated with a study of its materia medica (1966) et qui signe la même année The Book of Poisons of Ibn Wahshiya and its Relation to Indian and Greek Texts (1966) traduit du Kitab as-Sumum d'Ibn Wahsiya, lequel l'aurait lui-même dicté en arabe à Ali as Zayyat à partir d'un original en nabatéen. La version syriaque abrégée que Barhebraeus a donnée au XIII ${ }^{\mathrm{e}}$ s. du Kitab al-Adwiya (Livre des drogues simples) du médecin cordouan Ahmad al-Ghafiqi (XII ${ }^{\mathrm{e}}$ s.) est mise en anglais par Max Meyerhof et G. P. Sobhy sous le titre The Abridged Version of the Book of Simple Drugs of Ahmad Ibn Muhammad al-Ghafiqi by Gregorius abu l-Farag (Barhebraeus) (1932-1938) avec un lexique grec-latin-turc-persan-anglais-français-allemand. Myerhof, orientaliste d'origine allemande, traduit aussi en français le Kitab Sahr de Maïmonide, qui devient L'Explication des noms de drogues. Un glossaire de Matière médicale composé par Maïmonide, texte publié pour la première fois d'après le manuscrit unique, avec commentaire et index (1940). Le Kitab at-Tasrif d'Abulcasis trouve des interprètes en S. K. Hamarneh et G. Sonnedecker, qui publient conjointement A Pharmaceutical View of Abulcasis al-Zahrawi in Moorish Spain (1963). L'antidotaire du médecin cairote Ibn abi al-Bayan (1161-1240) est mis en français par C. Avierinos et P. Sbath et erronément attribué à Ibn Kaysan par l'intitulé Précis sur les médicaments employés dans la plupart des maladies par Sahlan Ibn Kaysan (1953); Otto Spies en donne une traduction partielle allemande en 1962. En Allemagne encore, Albert Dietrich signe Ein Arzneimittelverzeichnis des Abdul alLatif Ibn Yusuf al-Bagdadi (1967) traduit du traité de pharmacologie d'Abd al-Latif (1162-1231), médecin et philosophe à Bagdad.

Signalons pour terminer deux traductions du chinois: celle du Tchou-fan-tche, traité de matière médicale exotique composé au XII ${ }^{\mathrm{e}} \mathrm{s}$. par Tchao Jou-koua, due à la collaboration des Britanniques Hirth et Rockhill en 1911, et celle du Kiu-Lou (Traité de l'orange, 1178) de Han Yen-che, le plus ancien traité scientifique connu concernant les variétés de citrus, le seul de l'époque Song qui ait été traduit dans une langue occidentale (1923). 
Comme son titre l'indique, ce survol de la traduction pharmaceutique au cours des âges n'avait d'autre prétention que d'apporter sa modeste contribution à une facette particulière de l'histoire de la traduction. Il serait donc aisé de lui reprocher son caractère fragmentaire. C'est le reproche habituel adressé aux téméraires qui se lancent dans l'exploration de terres vierges. Mais il faut bien donner aux esprits critiques le prétexte à faire mieux.

\section{RÉFÉRENCES}

Boussel, P. (1949): Histoire illustrée de la pharmacie, Paris.

Bouvet, M. (1937) : «Histoire de la pharmacie», Histoire générale de la médecine, t. III, Paris.

Brandenburg, D. (1968): "Medizinisches im Avesta, der heiligen Schrift der Parsen", Medizinischer Monatsspiegel, 6, Darmstadt.

- (1969): Priesterärzte und Heilkunst im alten Persien, Stuttgart.

BRIM, C. J. (1936): Medicine in the Bible, New York.

Bryan, C. P. (1931): The Papyrus Ebers, New York.

Campbell, D. (1926): Arabian Medicine and its Influence in the Middle Ages, London.

CAP (1847): «Histoire de la pharmacie aux temps fabuleux et chez les Égyptiens, les Israélites, les Hindous et les Chinois", Journal de Pharmacie d'Anvers.

Chavy, P. (1988): Traducteurs d'autrefois, Genève.

Darmesteter, J. (1892-1893): Le Zend Avesta, traduction nouvelle avec commentaire historique et philologique, Paris.

Dubler, C. E. (1959) : «Die "Materia medica” unter den Muslimen des Mittelalters", Arch. Gesch. Medizin, 43.

— y E. Terés (1952-1957) : La versión árabe de la Materia medica de Dioscórides, TetuanBarcelona.

EbBell, B. (1939): The Papyrus Ebers, Copenhagen.

Elgood, C. (1951): A Medical History of Persia, Cambridge.

Fabre, R. et G. Dillemann (1974): Histoire de la pharmacie, Paris.

Farando de St. Germain, L. (1943): El Libre de les medicines particulars versión catalana trescentista del texto árabe del Tratado de los medicamentos simples de Ibn Wafid, autor médico toledano del siglo XI, Barcelona.

Finegan, J. (1959): Light from the Ancient Past, Princeton.

Gauthier, L. (1939): Antécédents gréco-arabes de la psycho-physique, avec le traité de posologie d'Al Kindi, Beyrouth.

Geldner, K. F. (1886-1896): Avesta. The Sacred Books of the Parsis, Stuttgart.

Huard, P. et M. Wong (1959): La médecine chinoise au cours des siècles, Paris.

Hubötter, F. (1957): Chinesisch-Tibetische Pharmakologie und Rezeptur, Ulm.

JACQ, C. (1981): L'Égypte des pharaons, Paris.

JonCKHeEre, F. (1947) : Le papyrus médical Chester Beatty, Bruxelles.

— (1955): "Les préparations et remèdes dans l'organisation de la pharmacie égyptienne», Egyptologische Studien.

Leake, C. D. (1952): Old Egyptian Medical Papyri, Lawrence.

LeCA, A.-P. (1971): La médecine égyptienne au temps des pharaons, Paris.

Martí-Ibañez, F. (1959): The Epic of Medicine, New York.

- (1961): A Prelude to Medical History, New York.

Meyerhof, M. (1935): «Esquisse d'histoire de la pharmacologie et botanique chez les musulmans d'Espagne», El Andalus, 3.

- (1940): Un glossaire de matière médicale composé par Maïmonide. Texte, traduction et commentaire, Le Caire. 
— (1941): «Études de pharmacologie arabe tirées des manuscrits inédits. III. Deux manuscrits illustrés du Livre des simples d'Ahmad al-Gafiqi. IV. Le recueil de descriptions de drogues simples du chérif al-Idrisi », Bull. Inst. d'Égypte, 23.

Millás-Vallicrosa (1943): «Un texto hebráico de la obra sobre los medicamentos simples de Ibn Wafid», Sefarad, 3.

Mosig, A. und G. Schramm (1955): Die Arzneipflanzen und der Drogenschatz Chinas, Berlin.

Percheron, D. et M. Le Roux (1955) : Petite histoire de la pharmacie, Avignon.

Pomet, P. (1694): Histoire générale des drogues simples, Paris.

Regnault, J. (1902): Médecine et pharmacie chez les Chinois, Paris.

Reutter de Rosemont, L. (1931): Histoire de la pharmacie à travers les âges, Paris.

Roberts, E. (1931): Vegetable Materia Medica of India and Ceylon, Colombo.

Ruska, J. (1932): «Arabische Giftbücher. I. Das Giftbuch des Inders Schanaq. II. Das Giftbuch des Gabir Ibn Hajjan. III. Die Gifte im Kanon des Avicenna », Fortschritte der Medizin, 50.

Sватн, P. (1937): "Traité sur les substances simples aromatiques par Yohanna ben Massawaih, grand savant et célèbre médecin chrétien décédé en 857 », Bull. Inst. d'Égypte, 19.

Schall, P. (1965): Zaubermedizin im alten China, Stuttgart.

Schelenz, H. (1904): Geschichte der Pharmazie, Berlin.

Schмidt, A. (1924): Drogen und Drogenhandel im Altertum, Leipzig.

Schramm, G. (1956): "Heilpflanzen und Drogen der altchinesischen Materia Medica", Forschung und Fortschritt, 30.

SIGGel, A. (1956) : «Medizin und Hygiene im Königsbuch des iranischen Dichters Firdausi », Die Medizinische Welt, 30.

— (1957) : «Al-Kindis Schrift über die zusammengesetzten Heilmittel», Arch. Gesch. Medizin, 37.

Sournia, J.-C. (1991): Histoire de la médecine et des médecins, Paris.

Spies, O. (1968): "Das türkische Drogen- und Medizinbuch des Ishaq Ibn Murad», Wissenschaftliche Zeitschrift der Martin-Luther-Universität, 17-2-3, Halle.

Steinschneider, M. (1866) : «Constantins Liber de gradibus und Ibn al Jazzars Adminiculum », Virchows Arch. für pathol. Anatomie, 37.

— (1873) : "Gifte und ihre Heilung, eine Abhandlung des Moses Maimonides », Virchows Arch. für pathol. Anatomie, 57.

— (1879): «Gafiki’s Verzeichnis einfacher Heilmittel», Virchows Arch. für pathol. Anatomie, 77.

- (1893): Die hebräischen Ueberstzungen des Mittelalters und die Juden als Dolmetscher, Berlin.

- (1956): Die europäischen Uebersetzungen aus dem Arabischen bis Mitte des 17. Jahrhunderts, Graz.

- (1960): Die arabischen Uebersetzungen aus dem Griechischen, Graz.

STERnon, F. (1933): Quelques aspects de l'Art pharmaceutique et du Médicament à travers les âges, Liège.

Thompson, C. J. S. (1928): The Mystery and Art of the Apothecary, London.

Thompson, R. C. (1923): Assyrian Medical Texts, London.

Ullmann, M. (1970): Die Medizin im Islam, Leiden.

VAN Hoof, H. (1993) : Dictionnaire universel des traducteurs, Genève.

Volger, L. (1941): Der Liber fiduciae de simplicibus medicinis des Ibn al-Jazzar in der Uebersetzung von Stephanus de Saragossa, Berlin.

WALlNöFer, H. (1966): Wissen vom langen Leben. Indische Heilkunst, Stuttgart.

— und A. von Rottauscher (1959): Der goldene Schatz der chinesischen Medizin, Stuttgart.

Wong, M. (1968) : "Contribution à l'histoire de la pharmacie traditionnelle chinoise», Revue d'histoire de la pharmacie, 19, p. 197-198. 